Indonesian Journal of Physics

Vol 19 No. 2, April 2008

\title{
Global innovation program for nanoscience education -How can we use electrons for our future, Hydrogen society-
}

\author{
Mitsuomi Yamaguchi, Mitsugu Hamasaki, Masumi Obara, \\ Kenji Watanabe*, and Kozo Obara \\ Dept. of Nanostructure and Advanced Materials, Kagoshima University, \\ Korimoto 1-21-40, Kagoshima 890-0065, Japan \\ kozo@eee.kagoshima-u.ac.jp \\ *Hydrogen Energy Laboratory Project U.S.A. INC. \\ 250 S. San Fernando Blvd. \#324 Burbank. CA 91520 \\ watanabe@haw-system.jp
}

\begin{abstract}
The change of global climate suggests the requirements of new technologies, which can suppress or improve the atmospheric composition. In this paper, we show a revolutionary car technology to decrease $\mathrm{CO}_{2}$. The key is the change of the fuel, from gasoline to hydrogen $+\mathrm{H}_{2} \mathrm{O}$. The fuel revolution generates hydrogen economy and induces new scientific targets. Nanoscience and nanotechnology are most expectable tools. Especially, understanding the varieties of characteristics of materials in electronic level is the most important therma. We propose international collaborations on nanoscience education to promote our knowledge and understanding each other.
\end{abstract}

Keywords : Revolutionary car technology, Decrease $\mathrm{CO}_{2}$, Hydrogen economy, Nanoscience and nanotechnology

\section{Introduction}

The chemical composition of Earth's atmosphere is changing because of the increased combustion of fossil fuels in the industrialized world. Recently, IPCC ${ }^{1)}$ reported the present situation of the Earth, in which the increase of the temperature by a few degrees drastically changes our circumstance. Although the predictions of the reports include many problems to be discussed, it suggests two important concepts; suppression of $\mathrm{CO}_{2}$ concentration and the development of new renewable energy.

In this paper, firstly, we present a new type hydrogen internal combustion engine, whose fuel is hydrogen and water ${ }^{2)}$. This technology is a most effective key to improve the greenhouse effect. One of the authors (K. Watanabe) developed the prototype of this advanced car in 1985. Many efforts developing the technologies to control the new type engine have been done by him, then, the large-scale production of the new type hydrogen cars starts at July $2008^{3}$.

Watanabe, a native of Kagoshima, the southernmost city of the island of Kyushu, has spent over 25 years developing the hydrogen+water engine system without government and institutional support. The essential his idea was to combine a hydrogen engine and a steam engine. Some demerits of hydrogen as the fuel of internal combustion engine are perfectly compensated by the characteristics of water mist (cluster).

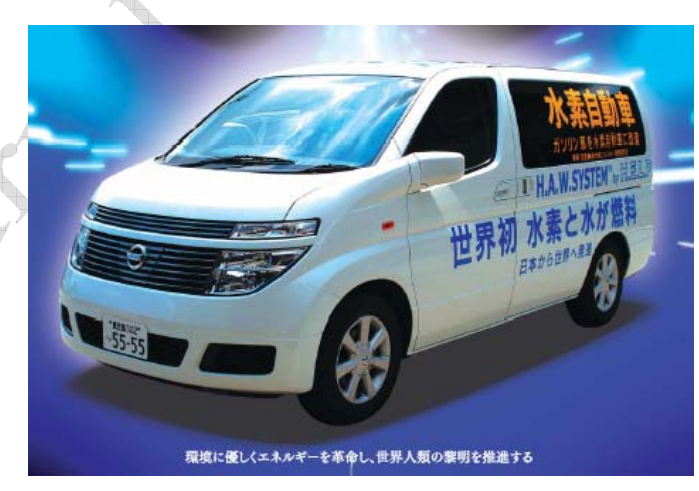

Figure 1. New type hydrogen car, which uses hydrogen and water as the fuel.

The processes of the combustion of hydrogen and water include many unknown area; atom and molecules reaction processes in high-pressure plasma, nitrogen oxide control in the combustion, and protection of hydrogen diffusion to the cylinder material. These are examples for simple cases. The really complex examples are directly related to the global system.

The recent arguments on hydrogen economy suggest the importance of molecular reactions in stratosphere ${ }^{4)}$. The stratosphere contains ozone layer, which affects the energy balance of the lower atmosphere. Circulation in the lower stratosphere affects tropospheric weather and climate.

Microscopic processes in the space from the atmosphere to the stratosphere contain the all processes in the new type hydrogen+ water engine. Therefore, the understanding of hydrogen+water engine promotes the 
understanding of the influences of greenhouse effects in the near future.

Secondary, we demonstrate some dynamic processes of water droplets on hot water surface ${ }^{5)}$. The evaporation process of $\mathrm{H}_{2} \mathrm{O}$ from water surface causes energy and momentum transfers between them. The tiny temperature deviation induces the movement of water mist. The collective movements of water mist show the similar behaviors of clouds in the sky. In these processes, contributions of electromagnetic effects are not clear in spite of the popular phenomena as the thunders and lighting. The electronic phenomena are the outputs of each reaction process in which electrons contribute largely. Existence of water mist layer on the water surface suggests the metastable layer between the gas phase and the liquid phase.

Thirdly, we discuss the generality of the surface phase as a metastable phase which exist between solid (or liquid ) phase and gas phase ${ }^{6}$. The characteristics of the surface phase dominate the growth process of condensed materials, energy dissipation processes, and diffusion processes of adsorbed materials on the substrate surface. In vapor phase growth processes as sputtering, the thickness of the surface phase is about one nanometer. The particle density profile and velocity profiles at the surface should be continuous. Existence of surface phase is one concept to connect with the dynamics or thermo dynamical parameters of condensed atoms and vapor phase.

Fourthly, electronic parameters are very important keys for collision processes. The most key is the contribution of electronic energy in the energy conservation rule, in which total energy is equal to the summation of Ee (electronic energy), Er (energy of rotation), and Ev (energy of vibration ). In this experiment we show the electronic energy dependence of crystal structures, electronic structures, and surface morphologies of condensed zinc thin films.

Finally, we propose the international collaborations on nanoscience and nano- technology areas of new materials for young generations.

\section{Change from Carbon Economy to Hydrogen Economy}

New systems using hydrogen are the most effective technology for expecting the decrease of greenhouse effect of the earth. However, we have many questions to solve the unknown situation of the global weather system ${ }^{7}$.

It is now widely accepted that the increased combustion of fossil fuels since the industrialization of the Western world has led to unprecedented changes in the chemical composition of Earth's atmosphere, with multiple consequences for regional air quality and the global climate system.

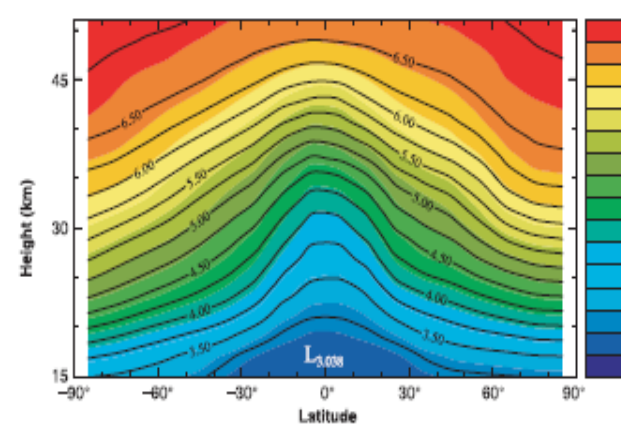

Figure 2. The background $\mathrm{H}_{2} \mathrm{O}$ mixing ratio (given by contours in units of ppmv) and the increase of stratospheric $\mathrm{H} 2 \mathrm{O}$ in January due to the assumed fourfold increase of $\mathrm{H} 2$, computed using the Caltech/JPL 2-D model (given by color in \% change). Refer from SCIENCE VOL 30013 JUNE 2003, 1741.

\section{Understanding of Behaviors of Water Molecules from Small Experimental System.}

We propose effective experimental system for understanding the real system of the earth. The characteristics of water vapor on the earth are very similar to the characteristics on your hot coffee cup. Cloud like white dusty region on the surface of coffee depends on the very small temperature difference on the water surface. The movement of water droplets is perfectly two- dimensional movement. Therefore, the movement of water droplet system reflects thermodynamic conditions including microscopic collisions of water molecules ${ }^{5)}$.

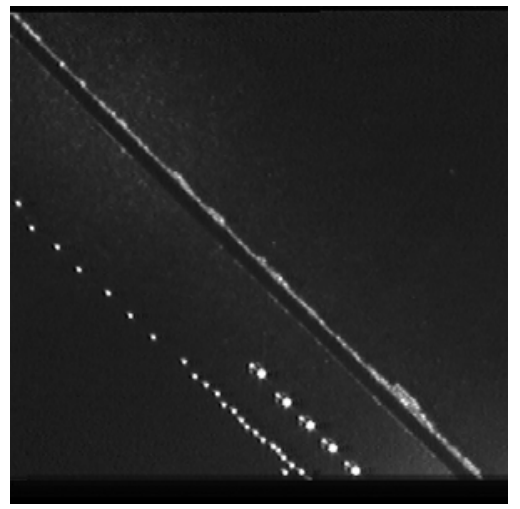

Figure 3. Typical example of movement of water droplets, whose diameter is $20-50 \mu \mathrm{m}$. In this photograph, the diameter of of platinum line heater is $50 \mu \mathrm{m}$. Temperature of background water surface is $70{ }^{\circ} \mathrm{C}$.

The droplet has attractive and repulsive forces generated by thermohydrodynamic effects. These droplets showed behaviors as a perfect two-dimensional particle system. By use of thin line heater, one-dimensional droplet array was formed. From the analysis of the distance between droplets in the array, temperature difference of the droplets and the maximum length of the thermohydrodynamic potential 
were derived. From condensation process to the one-dimensional array, the potential energies of the droplets were derived. The correlated motions of the droplets were observed when the structure of the droplet system transforms from one line to two lines. At least three droplets in the end of the array act as "boundary layer" in the condensation process.

The droplet has attractive and repulsive forces generated by thermohydrodynamic effects. These droplets showed behaviors as a perfect two-dimensional particle system. By use of thin line heater, one-dimensional droplet array was formed. From the analysis of the distance between droplets in the array, temperature difference of the droplets and the maximum length of the thermohydrodynamic potential were derived. From condensation process to the one-dimensional array, the potential energies of the droplets were derived. The correlated motions of the droplets were observed when the structure of the droplet system transforms from one line to two lines. At least three droplets in the end of the array act as "boundary layer" in the condensation process.

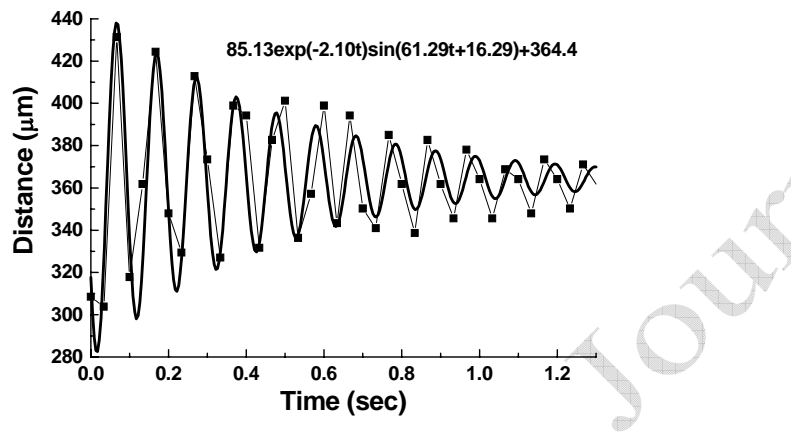

Figure 4. Typical data of vibration of water droplet which exist near platinum line heater. The decrease of kinetic energy suggests the increase of local "temperature" of water system. Time dependence of CCD camera images show real characteristics which we have to consider.

\section{Contribution of Electronic States of Surface Phase.}

Every Material consist of atoms and every materials does electrons and protons. In the processes of condensation or evaporation of materials, these two charged particles do essential contribution for these processes. Electron bombardments of the material surface modify the physical characteristics of the surface.

In metallic materials, electron bombard- ments is not effective because of rapid compensation process due to free electrons ${ }^{8)}$. The states of electrons strongly depend on the atomic distance. The correlations between incident electron energy, crystal lattice structure, and electron states of material surface are very important when we proceed the understanding of growth processes of advanced materials.

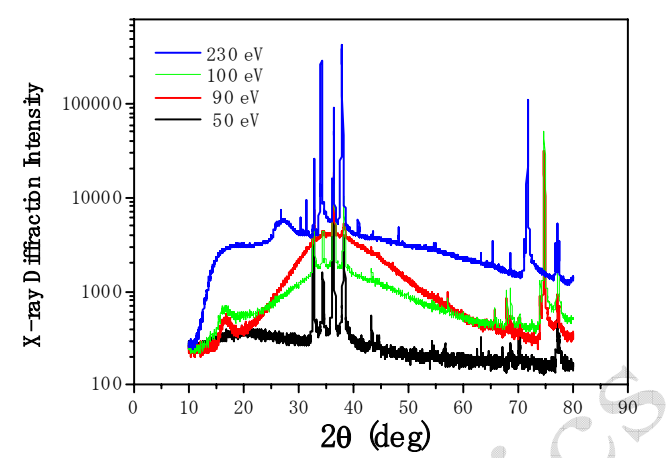

Figure 5. Incident electron energy dependence of X-ray diffraction intensities. Diffuse scattering intensities increased at $90 \mathrm{eV}, 100 \mathrm{eV}$ and $230 \mathrm{eV}$.

In this session, we present the mechanism of defect formation in metallic element, $\mathrm{Zn}$, induced by the electron irradiation. So far, there is no report, which shows the low energy electron irradiation damages in metallic film because the excited states in metallic substance immediately recombined with free electrons in it. These results, however, are all in post irradiation effect. Since the electron states are a function of the distance between two atoms, if we expect the observation of the electronic excitation states of metallic elements, it is essentially important to irradiate the isolated or separated metallic element by the electrons with appropriate energy. These situations are possible in the near surface region of the growth front.

The zinc films were deposited on insulative substrates in $\mathrm{P}<10^{-7}$ Torr under the electron irradiation. The incident angles of zinc atoms and electrons are normal and 45 deg to the surface of the sapphire substrate, respectively. The substrate surface was electrified by the incident electrons with $\mathrm{E}<240 \mathrm{eV}$ before Zn deposition.

Main results are followings; 1 ) electron energy dependence of crystal structure showed apparent increase of the diffuse scattering of X-ray intensity at $10,90,100,230 \mathrm{eV}$, which are related to the electron energy levels, $3 d(10 \mathrm{eV}), 3 p(90 \mathrm{eV}), 3 d+3 p(10+90$ $\mathrm{eV}), 3 \mathrm{~d}+3 \mathrm{~s}(90+140 \mathrm{eV})$, of zinc atoms, respectively. The transition at $140 \mathrm{~b} \mathrm{eV}$ from $3 \mathrm{~s}$ to $4 \mathrm{~s}$ is restricted due to the selection rule, 2) main peak energies of the electronic structure of deposited zinc films shifted by $12-15 \mathrm{eV}$ in 3s, 3p, 3d states at the incident energy 100 $\mathrm{eV}$ and $230 \mathrm{eV}$ only. These energy shifts mean the increase of the binding energy, not the satellites. 


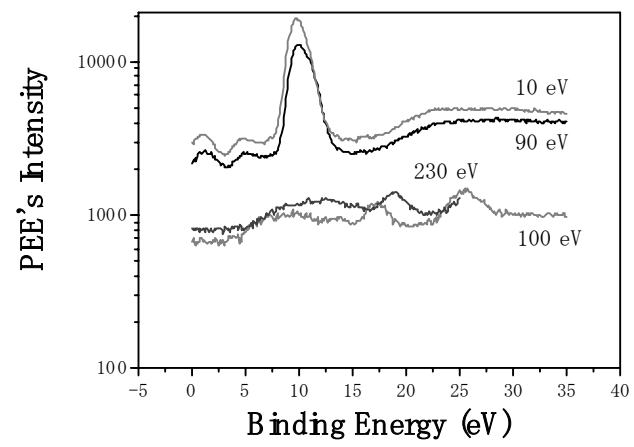

Figure 5. Incident electron energy dependence of $\mathrm{Zn}$ 3d-state. Main peaks of 3d shifted to higher energy by $15 \mathrm{eV}$ at $100 \mathrm{eV}$ and $230 \mathrm{eV}$.

In this session, we present the existence of zinc lattice defects with excited d-states induce by electron irradiation. There are two important keys for the observation of zinc atoms with excited d-states, that is, making holes in the d-states; one is the electron localization on the insulative substrate, and the another is the existence of very first quenching processes for the excited atoms.

For the electron localization, the special sapphire substrates, whose surfaces were covered with $\mathrm{Au}$ thin film except for the area of zinc deposition, were prepared. The Au thin film acts as the acceleration electrode of incident electrons. The incident electron energy is controlled by the supplied positive potential energy $\mathrm{eV}_{\mathrm{p}}$ of the $\mathrm{Au}$ electrode. The incident electrons electrify the substrate surface for zinc deposition up to the incident electron energy. After charging the substrate surface, zinc atoms are evaporated from the zinc beam source.

The incident zinc atoms are ionized by the incident electrons before colliding the substrate surface. Since the polarity of the electric field on the substrate changes at the critical distance, $\mathrm{Zn}^{+}, \mathrm{Zn}^{2+}$ are selectively deposited on the negatively charged substrate surface. Finally, ionized zinc atoms interact with the localized surface electrons with potential energy, $\mathrm{eV}_{\mathrm{p}}$. In the final collision process at the surface, excited d-states of zinc atoms are formed due to Auger processes. The excited states are immediately quenched at the growth surface without recombination. The existence of holes in the d-states is confirmed by the increase of binding energy of zinc about $15 \mathrm{eV}$, which is comparable to the energy difference between $\mathrm{Zn}$ and $\mathrm{Ge}$, and enhancement of diffuse scattering of $\mathrm{X}$-ray diffraction intensity. Formed zinc films show insulative characteristics and the existence of magnetic interaction in them. This technique is very useful for advanced new spin electronics devices.

\section{Our Future International Collaboration on Nanoscience Education}

We have some plans to propose a new program for international collaborations on Nanoscience education and the future hydrogen society to our governments. These programs include international symposium for exchange with foreign students and researchers every year. The final purposes are 1) publication of text for Nanoscience education, 2) developments of educational kits for nanoscience and hydrogen power. These products will be powerful funds on hand for our future activities. If this proposition is accepted, we are able to promote our international collaboration greatly.

\section{References}

1. Contribution of Working Group I to the Fourth Assessment Report of the Inter- governmental Panel on Climate Change (2007), www.ipcc.ch.

2. Automotive Industries 1985 August pp.51-53.

3. http//www.haw-system.jp

4. M. P. Baldwin, M. Dameris, T. G. Shepherd, Science 316, 1577, 2007.

5. K. Obara , Y. Ohtera, and Y. Suemoto, J. Crystal Growth, 166, 136, 1996.

6. Yu Hailong, et al., The 9th International Symposium on Sputtering and Plasma Processes, Kanazawa 2007, TF P1-10, 1-4, 2007.

7. For example, www.sciencemag.org 10 OCTOBER VOL 302 SCIENCE, 226, and others in web of SCIENCE, 2007.

8. N. Itoh, Nuclear Instruments and Methods in Physics Research B , 135, 175-183, 1998. 